\title{
Local climate zone mapping of Tirana, Albania
}

\author{
Anja Cenameri ${ }^{\mathrm{a}}$, Gáspár Albert ${ }^{\mathrm{b}}$ \\ ${ }^{a}$ Eötvös Loránd University, Institute of Cartography and Geoinformatics, Budapest, Hungary, \\ anjacenameri@student.elte.hu \\ ${ }^{\mathrm{b}}$ Eötvös Loránd University, Institute of Cartography and Geoinformatics, Budapest, Hungary, albert@ludens.elte.hu
}

Keywords: Environmental mapping, Local climate zones, LCZ generator, Climate change, Urban planning

\begin{abstract}
:
Over the last thirty years, the capital city of Albania undergone radical changes and the demands of the population increased drastically. New districts are built and old ones are given a completely new character. Meanwhile the municipality of Tirana is increasingly suffering from environmental and climate problems, like many cities in the Mediterranean. Climate change observed globally has become even more severe in these areas, and environmental mapping is crucial in order to be prepared for the inevitable changes. In order to locate the most environmentally vulnerable neighbourhoods a Local Climate Zone mapping of Tirana was performed.

The mapping was done simultaneously on two scales: 1) a satellite data based analysis was done on the whole municipality of Tirana, and 2) a large scale analysis was done using detailed vector data like: building's database, road network, impervious and pervious surface, and canopy height. Some of the above-mentioned layers were obtained by analysing/processing the DSM and DTM from a chosen pilot area situated in the heart of the city. The medium scale map was compiled using the open source LCZ generator which works on Google Earth Engine. This is based on a supervised classification, for which we used 5-15 training areas for each LCZ types, according to the guidelines, because several examples of each LCZ type help in the automatic classification. The large scale map of recent urban climatic zones of the pilot area was compiled through GIS spatial queries using QGIS. In the case of the city of Tirana no such analyses were done yet, so the aim of this study was to perform a preliminary analysis on the state of the recent urban climate zones of the area.

The results show that $5.38 \%\left(5.78 \mathrm{~km}^{2}\right)$ of the total area of the municipality is categorized as compact midrise, while $4.84 \%\left(5.2 \mathrm{~km}^{2}\right)$ is classified as compact low-rise and a value of $2.3 \%\left(3.21 \mathrm{~km}^{2}\right)$ belongs to compact high-rise, referring to a total area of $107.3 \mathrm{~km}^{2}$, which includes almost one-tenth of the official administrative border of the municipality. While the results of large-scale mapping of the pilot zone, which includes an area of $4.09 \mathrm{~km}^{2}$, present as predominant in the value of $36.7 \%\left(1.5 \mathrm{~km}^{2}\right)$ compact low-rise, $24.7 \%\left(1.01 \mathrm{~km}^{2}\right)$ open low-rise and $10.27 \%\left(0.42 \mathrm{~km}^{2}\right)$ sparsely built.(Figure 1$)$. The detailed map of the pilot area shows more complex characteristics than the satellite image based LCZ map.
\end{abstract}

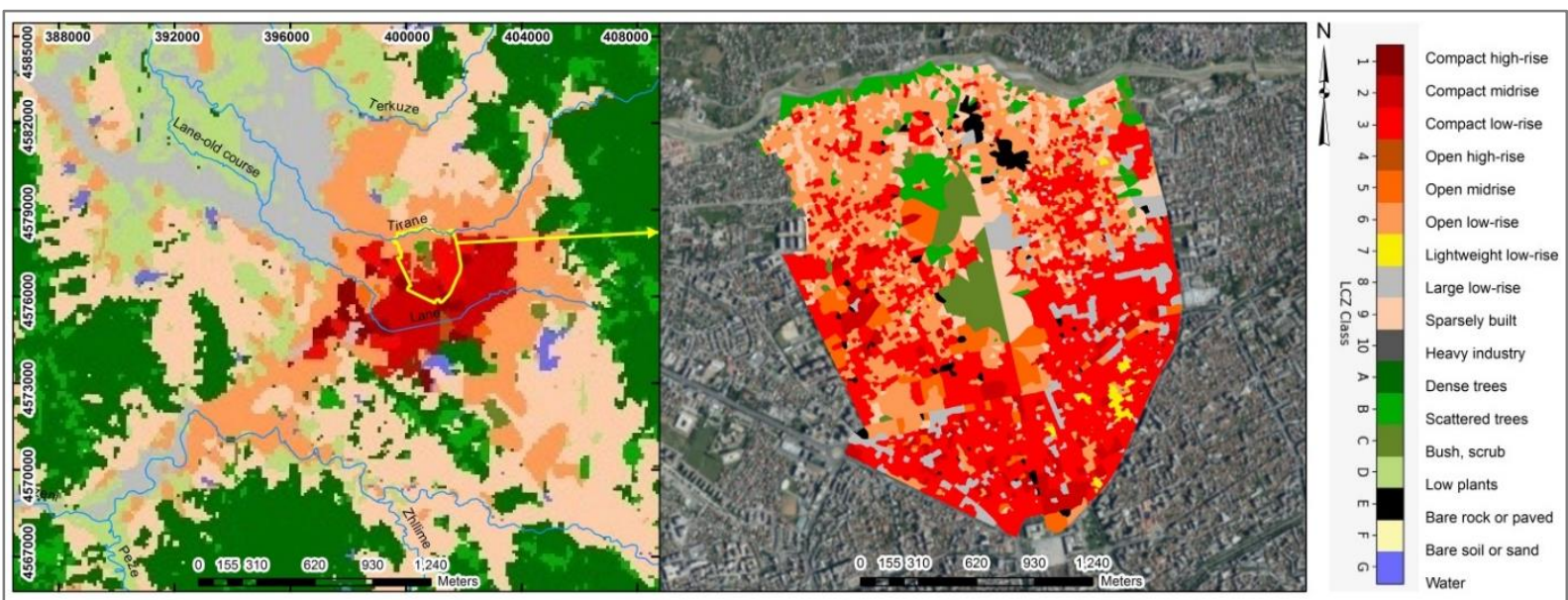

Figure 1. Local Climate Zone maps of Tirana municipality and its surroundings. The medium scale map on the right was compiled using the LCZ Generator. The large scale map on the left shows the pilot area compiled through GIS spatial queries. As base map, orthophotos from 2018 were used, which cover the area of TiranaDurres. The coordinate system is UTM Zone $34 \mathrm{~N}$ (WGS84). 
The characteristics of the local climatic zones are more distinct in the case of the detailed map of the pilot zone. On the other hand, the satellite image based LCZ map is a very good way to get an overview of the situation of the whole city or municipality, which can be used to identify the most problematic areas and then refer to them for more detailed studies.

We concluded that the large scale mapping is more effective on identifying the varying urban climate zones on the level of city planning. At this scale, the LCZ map can also help the responsible authorities to intervene to improve the situation through local regulatory plans. Reducing the urban heat island effect requires conscious urban planning, complemented by policies to raise citizens' awareness. We hope that our work can contribute to this process, resulting in more green spaces in the city centre and beyond. 